\title{
Parallel-Coupled Coplanar-Waveguide Bandpass Filter With Multiple Transmission Zeros
}

\author{
Chao-Huang Wu, Chi-Hsueh Wang, Yo-Shen Lin, and Chun Hsiung Chen, Fellow, IEEE
}

\begin{abstract}
A compact parallel-coupled coplanar-waveguide bandpass filter with good selectivity and stopband response is proposed. By using the quarter-wavelength resonators in implementation, the filter size can be reduced and no spurious passband is observed at twice the center frequency $\left(2 f_{0}\right)$. In addition, by suitably introducing the capacitive and inductive cross-coupling effects, four transmission zeros around the passband edges may be created for improving the filter selectivity. For design purpose, the equivalent-circuit models are also established. In this study, the compact fourth-order filter with multiple transmission zeros for improving the selectivity is implemented, and its stopband is extended up to $3.43 f_{0}$ with a rejection better than $-30 \mathrm{~dB}$.
\end{abstract}

Index Terms-Bandpass filter (BPF), coplanar waveguide $(\mathrm{CPW})$, coupled lines.

\section{INTRODUCTION}

$\mathbf{T}$ HE coplanar waveguide (CPW) structure is attractive as an alternative to the microstrip for planar filter designs due to its insensitivity to the substrate thickness. Various works on parallel-coupled CPW bandpass filters (BPFs) were reported in the literature [1]-[3]. In modern communication system applications, it is necessary to have the filters with very good selectivity for better image or interference rejection. To this end, the four-pole filter with two transmission zeros was reported in [4]. In [5], the quasi-elliptic CPW BPFs based on stepped-impedance resonators and capacitive cross-coupling effect were proposed to achieve good selectivity and compact size.

In addition to transmission zeros, an improvement of the stopband rejection is also important. Up to now, only a few of the CPW BPFs were proposed to suppress the spurious responses [6], [7]. The particular pattern of via holes was used in the double-surface CPW filter [6] to reduce the spurious responses. Another four-pole BPF was introduced in [7] using the two asymmetric parallel-coupled CPW stages at the input/output terminated ends to generate two transmission zeros for suppressing the harmonic passband around triple of the center frequency $\left(3 f_{o}\right)$. However, the CPW structures in [1]-[7] can not achieve compact size, good selectivity, and good stopband rejection at the same time.

Manuscript received July 13, 2006; revised November 8, 2006. This work was supported by the National Science Council of Taiwan, R.O.C., under Grants NSC 94-2752-E-002-001-PAE, NSC 94-2219-E-002-008, and NSC 94-2213-E002-055.

C. H. Wu, C. H. Wang, and C. H. Chen are with the Department of Electrical Engineering and Graduate Institute of Communication Engineering, National Taiwan University, Taipei 106, Taiwan, R.O.C. (e-mail: chchen@ew.ee.ntu.edu. tw).

Y. S. Lin is with the Department of Electrical Engineering, National Central University, Chungli 320, Taiwan, R.O.C.

Digital Object Identifier 10.1109/LMWC.2006.890334
In this work, a compact fourth-order parallel-coupled CPW $\mathrm{BPF}$, based on quarter-wavelength $(\lambda / 4)$ resonators, is proposed. By combining the parallel and antiparallel coupled-line sections in the filter design, along with the control of inherent transmission zeros associated with the coupled line sections, the proposed CPW filter may achieve compact circuit size with the transmission zeros to improve the filter stopband rejection. In addition, by suitably introducing the capacitive and inductive cross-coupling effects, four transmission zeros around the passband edges may be created to improve the filter selectivity. Comparing with the microstrip filter in [8], the proposed CPW structure does not require the via-holes to realize the inductors, therefore the implemented filter would be less sensitive to the fabrication process. In addition, an inductive cross-coupling may easily be realized by using the CPW structure, while the realization in microstrip structure [8] would be complicated. Although the bondwires are needed for ground plane equalization and an extra ground plane may sometimes be required for developing a grounded-CPW configuration, the proposed CPW filter still has the merit of less sensitivity to the fabrication process.

\section{FOURTH-ORDER BPF}

\section{A. Filter Structure and Equivalent-Circuit Models}

Fig. 1(a) shows the layout of the proposed fourth-order parallel-coupled CPW BPF based on the $\lambda / 4$ resonators. The corresponding circuit model is also shown in Fig. 1(b). Here, both capacitive and inductive cross-couplings, $C_{\text {cross }}$ and $L_{\text {cross }}$, are introduced so as to create four transmission zeros around passband edges for improving the filter selectivity. The filter structure is composed of the parallel coupled line for the third section and the anti-parallel coupled lines for the other sections. The capacitive cross-coupling $C_{\text {cross }}$ is realized by the gap-coupled capacitor structure between the open-ends of coupled-line sections, and the inductive cross-coupling $L_{\text {cross }}$ is realized by the mutual inductor structure between the shorted-ends of input/output coupled-line sections.

By neglecting the cross-couplings, i.e., $C_{\text {cross }}=L_{\text {cross }}=$ 0 , and assuming that the even- and odd-mode phase velocities are equal, the circuit model Fig. 1(b) of the filter structure in Fig. 1(a) for all frequencies may first be represented by the one in Fig. 2(a) [9, Sec. 5.09].

The input shorted-end coupled-line section of length $\theta_{1}$ in Fig. 1(b) may be modeled by an ideal transformer of turn ratio $N_{1}$ with a transmission line of characteristic admittance $Y_{\mathrm{s} 1}$ and a shunt shorted stub of characteristic admittance $Y_{\mathrm{p} 1}$ at its two ends as in Fig. 2(a). The coupled-line sections with open-ends 


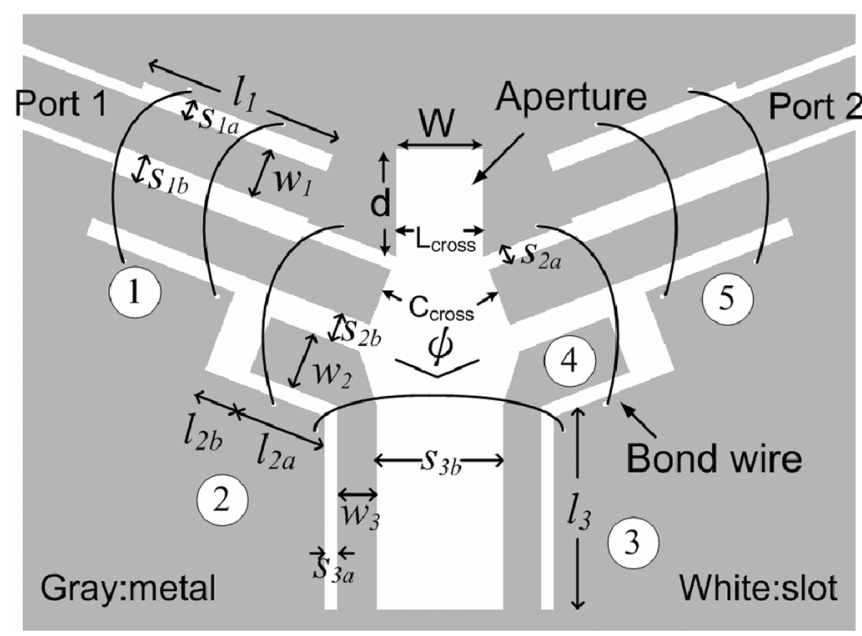

(a)

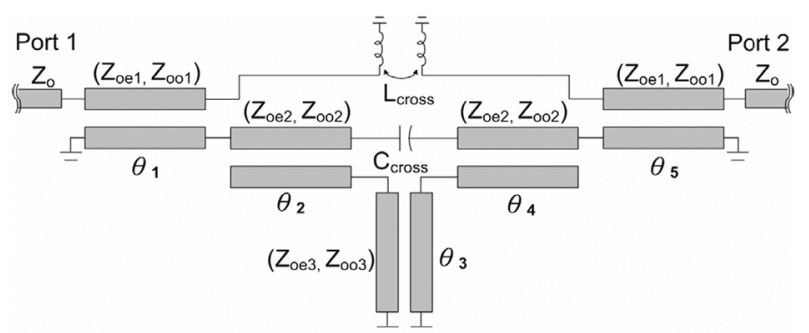

(b)

Fig. 1. Proposed fourth-order parallel-coupled CPW filter with capacitive and inductive cross-couplings: (a) layout and (b) circuit model. $\left(w_{1}=3.4 \mathrm{~mm}\right.$, $s_{1 \mathrm{a}}=0.5 \mathrm{~mm}, s_{1 \mathrm{~b}}=1.9 \mathrm{~mm}, l_{1}=14 \mathrm{~mm}, w_{2}=4 \mathrm{~mm}, s_{2 \mathrm{a}}=0.7 \mathrm{~mm}$ $s_{2 \mathrm{~b}}=2 \mathrm{~mm}, l_{2 \mathrm{a}}=6.5 \mathrm{~mm}, l_{2 \mathrm{~b}}=3 \mathrm{~mm}, w_{3}=1.4 \mathrm{~mm}, s_{3 \mathrm{a}}=0.2 \mathrm{~mm}$, $\left.s_{3 \mathrm{~b}}=11.2 \mathrm{~mm}, l_{3}=14 \mathrm{~mm}\right)$.

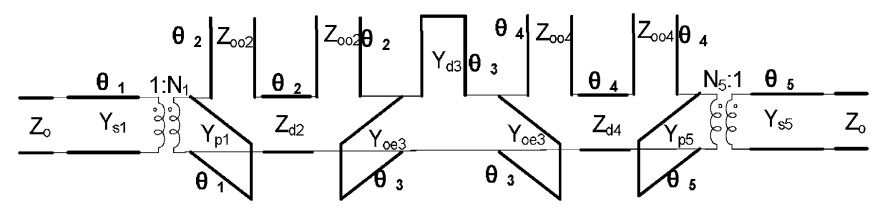

(a)

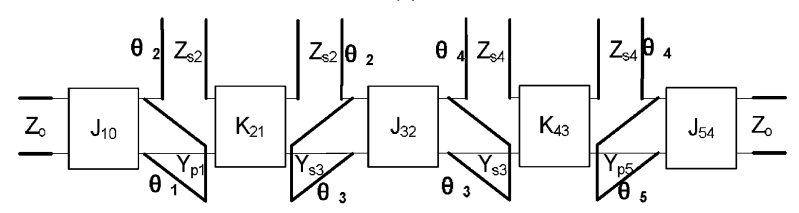

(b)

Fig. 2. Equivalent-circuit models for the proposed fourth-order parallel-coupled CPW BPF in Fig. 1 without the capacitive and inductive cross-couplings. The parameters are defined in (1) and (2).

of lengths $\theta_{2}$ may be modeled by a transmission line of characteristic impedance $Z_{\mathrm{d} 2}$ with two series open stubs of characteristic impedance $Z_{\mathrm{oo} 2}$ at its two ends. Similar modeling may also be applied to the coupled-line sections of lengths $\theta_{3}, \theta_{4}$ and $\theta_{5}$. Here, the design parameters are defined by

$$
\begin{aligned}
& N_{\mathrm{m}}=\left(Z_{\text {oem }}+Z_{\text {oom }}\right) /\left(Z_{\text {oem }}-Z_{\text {oom }}\right) \\
& Y_{\mathrm{pm}}=2 \cdot Y_{\mathrm{oem}} \cdot Y_{\mathrm{oom}} /\left(Y_{\mathrm{oem}}+Y_{\mathrm{oom}}\right) \\
& Y_{\mathrm{sm}}=\left(Y_{\mathrm{oem}}+Y_{\mathrm{oom}}\right) / 2 \quad m=1,5 \\
& Z_{\mathrm{dn}}=\left(Z_{\text {oen }}-Z_{\text {oon }}\right) / 2 \quad n=2,4 \\
& Y_{d 3}=\left(Y_{o o 3}-Y_{o e 3}\right) / 2 \text {. }
\end{aligned}
$$

The equivalent-circuit model in Fig. 2(a) reveals that when $\theta_{1}, \theta_{3}$, and $\theta_{5}=180^{\circ}$ at certain frequencies, the shunt shorted stubs in Fig. 2(a) will present low impedance to the ground such that no signal is coupled to the output port and a transmission zero will be formed. Thus the stub lengths $\left(\theta_{1}, \theta_{3}\right.$, and $\left.\theta_{5}\right)$ can be designed to suppress the spurious responses. Similarly, when $\theta_{2}$ and $\theta_{4}=180^{\circ}$ or $\theta_{3}=90^{\circ}$ at certain frequencies, the transmission zero will be observed due to the open circuit. Note that the equivalent-circuit model in Fig. 2(a) can easily predict the inherent transmission zeros of coupled line sections for suppressing the spurious responses.

For design propose, the equivalent-circuit model in Fig. 2(a) is modified to the one in Fig. 2(b) which is demonstrated as below. When the values of $Y_{\mathrm{sn}}(n=1,5)$ are equal to the reference admittance $Y_{o}\left[Y_{\mathrm{sn}}=\left(Y_{\mathrm{oen}}+Y_{\mathrm{oon}}\right) / 2=Y_{o}, n=1,5\right]$, the transmission lines of $\left(Y_{\mathrm{sn}}, \theta_{n}\right)$, the additional transmission line of $\left(Y_{o}, \theta / 2-\theta_{n}\right)$, and the ideal transformer in Fig. 2(a) may be combined to form the $J$-inverter $\left(J_{\mathrm{n}, \mathrm{n}-1}\right)$ as shown in Fig. 2(b). Besides, the series shorted stub of characteristic admittance $Y_{\mathrm{d} 3}$ with additional shunt shorted stub of characteristic admittance $\left(-Y_{d 3}\right)$ at its two ends may be modeled as the $J$-inverter $\left(J_{32}\right)$ [9, Sec. 8.03]. Thus, the middle parallel coupled-line section with shorted-ends in Fig. 2(a) may be modeled as the $J$-inverter $\left(J_{32}\right)$ with additional shunt shorted stub of characteristic admittance $Y_{\mathrm{s} 2}$ at its two ends Fig. 2(b). Similar modeling may also be applied to the coupled-line sections of lengths $\theta_{2}$ and $\theta_{4}$ [10]. Here, the design parameters are

$$
\begin{aligned}
J_{\mathrm{m}, \mathrm{m}-1} & =\left|\left(Y_{\mathrm{oom}}-Y_{\mathrm{oem}}\right) / 2\right| \quad m=1,5 \\
K_{\mathrm{n}, \mathrm{n}-1} & =\left|\left(Z_{\mathrm{oen}}-Z_{\mathrm{oon}}\right) /\left(2 \times \sin \theta_{n}\right)\right| \quad n=2,4 \\
Y_{s 3} & =\left(Y_{o e 3}+Y_{\text {oo } 3}\right) / 2 \\
J_{32} & =\left|\left(Y_{o o 3}-Y_{o e 3}\right) \times \cot \theta_{3} / 2\right| .
\end{aligned}
$$

Thus, by combining the above equivalences, one may finally yield the equivalent-circuit model as shown in Fig. 2(b).

\section{B. Filter Implementation and Results}

The proposed filter structure is fabricated on the Rogers RO4003c substrate $\left(\varepsilon_{r}=3.38, \tan \delta=0.0023\right.$, and thickness $h=1.524 \mathrm{~mm}$ ). The CPW BPF for Fig. 1(a) is designed with a center frequency $f_{o}$ of $1.78 \mathrm{GHz}$, a $3 \mathrm{~dB}$-bandwidth of $10 \%$, and a reference impedance $Z_{o}$ of $50 \Omega$. The corresponding circuit parameters, based on the equivalent-circuit model in Fig. 2(b), are as follows: $Z_{0 e 1}=Z_{0 e 5}=63.6 \Omega, Z_{o o 1}=Z_{o o 5}=37.4 \Omega$, $Z_{0 e 2}=Z_{0 e 4}=52.4 \Omega, Z_{o o 2}=Z_{o o 4}=44.3 \Omega$, $Z_{\text {oe } 3}=54.2 \Omega, Z_{o o 2}=46.8 \Omega, \theta_{1}=\theta_{3}=60^{\circ}, \theta_{2}=30^{\circ}$ at $f_{0}$.

In the layout of the proposed fourth-order CPW BPF Fig. 1(a), the coupled-line sections are bent to an angle $\psi$ for achieving larger capacitive and inductive cross-couplings. The aperture (width $w$ and length $d$ ) in Fig. 1(a) is used to decrease the inductive cross-coupling.

The locations of the cross-coupled-induced transmission zeros may actually be adjusted by varying the parameter $\psi$ Fig. 1(a) to control the capacitive and inductive cross-couplings and the parameter $d$ to control the inductive cross-coupling. The fullwave simulated responses (using the Ensemble simulator) of the proposed filter with respect to the parameters $\psi$ and $d$ 


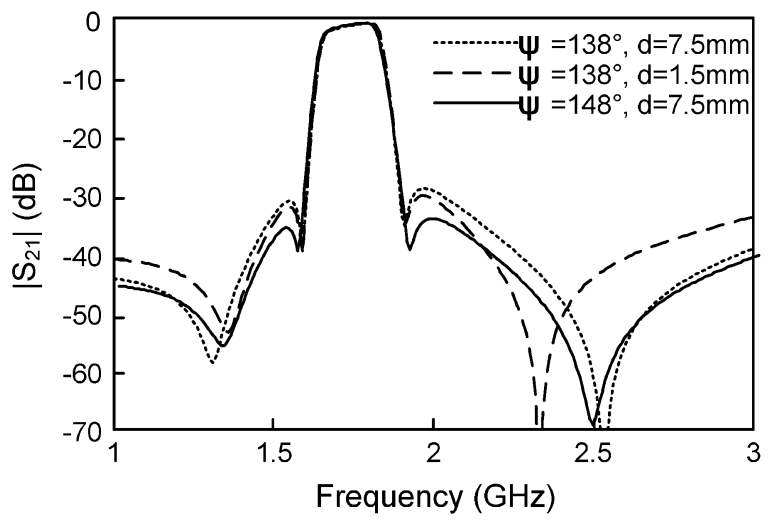

Fig. 3. Fullwave simulated responses of the proposed filter in Fig. 1(a) with $\psi$ and $d$ as parameters $(w=6.2 \mathrm{~mm})$.

in Fig. 1(a) are shown in Fig. 3. As demonstrated in Fig. 3, the inner transmission zeros move toward the center frequency $\left(f_{o}\right)$ and the outer transmission zeros move away from the center frequency as the parameter $\psi$ is decreased to increase the capacitive and inductive cross-couplings. And the outer transmission zeros will move toward the center frequency and the inner transmission zeros are only slightly changed as the parameter $d$ is decreased to increase the inductive cross-coupling. Specifically, based on the specified fall-off rate at the passband edge, one may determine the required $\psi$ and $d$ values for the desired locations of cross-coupled-induced transmission zeros. The controllable frequency ranges of the inner transmission zeros associated with the filter in Fig. 1 are from the lower passband edge $1.67 \mathrm{GHz}$ to $1.58 \mathrm{GHz}$ and the upper passband edge $1.83 \mathrm{GHz}$ to $1.87 \mathrm{GHz}$. The ranges of the outer ones are from the zero frequency to $1.58 \mathrm{GHz}$ and from $1.87 \mathrm{GHz}$ to $4.1 \mathrm{GHz}$.

The simulated results by the fullwave simulator Ensemble and the measured ones of the filter in Fig. 1(a) are shown in Fig. 4. The implemented filter has a size of $0.37 \lambda \times 0.33 \lambda$ (45.5 $\mathrm{mm} \times 40.6 \mathrm{~mm}$ ), where $\lambda$ is the guided wavelength of the $\mathrm{CPW}$ structure at center frequency. The measured center frequency is at $1.75 \mathrm{GHz}$. The minimum measured insertion loss is $2.15 \mathrm{~dB}$ at $1.75 \mathrm{GHz}$, and the $3-\mathrm{dB}$ bandwidth is $9.16 \%$. Four cross-coupled-induced transmission zeros are observed at $1.26 \mathrm{GHz}, 1.6 \mathrm{GHz}, 1.88 \mathrm{GHz}$, and $2.54 \mathrm{GHz}$, respectively. Here, the coupled-line sections $\left[\theta_{1}\right.$ and $\theta_{5}$ in Fig. 2(a)] have the inherent transmission zeros around $4 \mathrm{GHz}$ and the coupledline section $\left[\theta_{3}\right.$ in Fig. 2(a)] has the inherent transmission zero around $5 \mathrm{GHz}$. Specifically, the stopband rejection is better than $-30 \mathrm{~dB}$ up to $6 \mathrm{GHz}\left(3.43 f_{0}\right)$.

\section{CONCLUSION}

In this study, the compact fourth-order parallel-coupled CPW BPF based on $\lambda / 4$ resonators has been proposed and carefully examined. By the alternative connection of open-end and

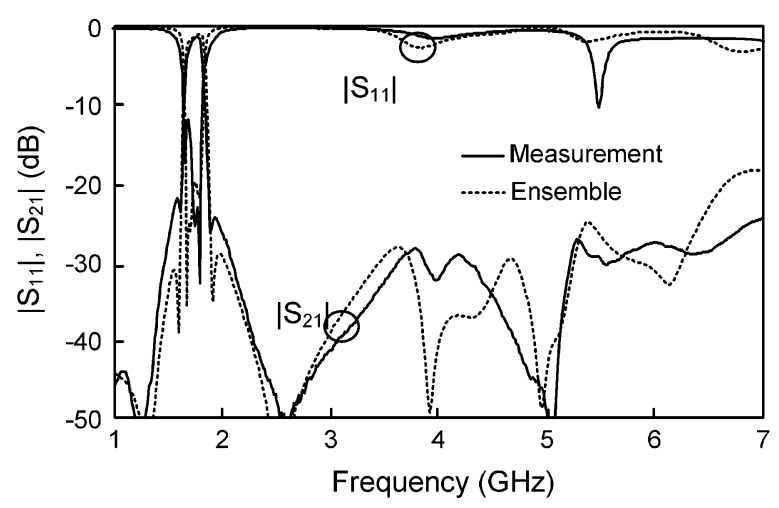

Fig. 4. Measured and simulated responses for the proposed fourth-order parallel-coupled CPW BPF shown in Fig. 1 (a). $(w=6.2 \mathrm{~mm}, d=1.5 \mathrm{~mm}$, $\psi=138^{\circ}$ ).

shorted-end coupled-line sections, the proposed BPF exhibits the merits of reduction in size and suppression of spurious passband around $2 f_{0}$. The lengths of coupled-line sections can be adjusted to improve the stopband rejection. Based on the proposed equivalent-circuit models, one may implement the desired filter with good selectivity and stopband response. Specifically, the fourth-order BPF with six transmission zeros have been designed and its stopband rejection is found better than $-30 \mathrm{~dB}$ up to $3.43 f_{0}$. The proposed filter is useful for applications in the communication systems when high selectivity and good stopband rejection are required.

\section{REFERENCES}

[1] S. Usyal, "Coplanar waveguide edge-coupled bandpass filters with finite ground planes," Electron. Lett., vol. 33, no. 5, pp. 375-376, Feb. 1997

[2] F.-L. Lin, C.-W. Chiu, and R.-B. Wu, "Coplanar waveguide bandpass filter-a ribbon-of-brick-wall design," IEEE Trans. Microw. Theory Tech., vol. 43, no. 7, pp. 1589-1596, Jul. 1995.

[3] T. M. Weller, "Edge-coupled coplanar waveguide bandpass filter design," IEEE Trans. Microw. Theory Tech., vol. 48, no. 12, pp. 2453-2458, Dec. 2000.

[4] T. Tsujiguchi, H. Matsumoto, and T. Nishikawa, "A miniaturized end-coupled bandpass filter using $\lambda / 4$ hairpin coplanar resonators," in IEEE MTT-S Int. Tech. Dig., June 1998, pp. 829-832.

[5] J. Zhou, M. J. Lancaster, and F. Huang, "Coplanar quarter wavelength quasi-elliptic filters without bond-wire bridges," IEEE Trans. Microw. Theory Tech., vol. 52, no. 4, pp. 1150-1156, Apr. 2004.

[6] T. Tsujiguchi, H. Matsumoto, and T. Nishikawa, "A miniaturized double-surface CPW bandpass filter improved spurious responses," IEEE Trans. Microw. Theory Tech., vol. 49, no. 5, pp. 879-885, May 2001.

[7] J. Gao and L. Zhu, "Asymmetric parallel-coupled CPW stages for harmonic suppressed $\lambda / 4$ bandpass filters," Electron. Lett., vol. 40, no. 18, pp. 1122-1123, Sep. 2004.

[8] C.-H. Wu, Y.-S. Lin, C.-H. Wang, and C. H. Chen, "Compact microstrip coupled-line bandpass filter with two cross-couplings for creating multiple transmission zeros," in Proc. 35th Eur. Microw. Conf., Paris, France, Oct. 2005, pp. 1267-1270.

[9] G. L. Mattaei, L. Young, and E. M. T. Jones, Microwave Filters, Impedance-Matching Networks, and Coupling Structures. Norwood, MA: Artech House, 1980.

[10] C.-M. Tsai, H.-M. Lee, and C.-C. Tsai, "Planar filter design with fully controllable second passband," IEEE Trans. Microw. Theory Tech., vol. 53, no. 11, pp. 3429-3439, Nov. 2005. 